\title{
Optimal reference states for maximum accessible entanglement under the local particle number superselection rule
}

\author{
G.A. White, J.A. Vaccaro, and H.M. Wiseman \\ Centre for Quantum Computer Technology, Centre for Quantum Dynamics, \\ School of Science, Griffith University, Brisbane, 4111 Australia
}

(Dated: January 19, 2009)

\begin{abstract}
Global conservation laws imply superselection rules (SSR) which restrict the operations that are possible on any given state. Imposing the additional constraint of local operations and classical communication (LOCC) forbids the transfer of quantum systems between spatially separated sites. In the case of particle conservation this imposes a SSR for local particle number. That is, the coherences between subspaces of fixed particle number at each site are not accessible and any state is therefore equivalent to its projection onto these subspaces. The accessible entanglement under the SSR is less than (or equal to) that available in the absence of the SSR. An ancilla can be used as a reference system to increase the amount of accessible entanglement. We examine the relationship between local particle number uncertainty and the accessible entanglement and consider the optimal reference states for recovering entanglement from certain systems. In particular we derive the optimal ancilla state for extracting entanglement for a single shared particle and make steps towards the optimum for general systems. We also show that a reference for phase angle is fundamentally different to a reference for the SSR associated with particle conservation.
\end{abstract}

PACS numbers: 03.67.-a, 03.67.Mn, 11.30.-j, 03.65.Ta

\section{INTRODUCTION}

Some five decades ago Wick, Wightman and Wigner showed that conservation laws induce so called "superselection rules" (SSR) that forbid the observation of coherences between different eigenstates of the conserved quantity by any physical measurement [1]. A decade later Aharanov and Susskind demonstrated that this restriction can be alleviated with an additional system that acts as a reference for the coherences [2]. Such reference ancillae are of particular interest when the dimension of the Hilbert space it occupies is not arbitrarily large compared to that of the system. In such cases the quantum nature of the reference system is important. We refer to such a reference system as a finite reference because of the finite dimension of the reference system's Hilbert space [36]. This is the regime that we are mainly concerned with here.

There has been some laxity in the use of terms to distinguish the classical and quantum regimes in the literature. To avoid any possible confusion we shall reserve the term reference frame for a set of independent variables which, in principle, can be defined without an explicit relation to any physical object. The inertial reference frames in special relativity are an example. In contrast, we use the terms reference system and reference ancilla to describe a physical system whose observables are used as a reference for other physical systems. This distinction between frames and systems is crucial in theories such as quantum gravity where external reference frames are artificial and a description of a physical system can only be made relative to other physical systems [3, 4].

The lack of the ability to observe coherences under a SSR implies that there are operations whose effect on the system are not physically detectable. Conversely, the ex- istence of such operations implies that a SSR operates. This converse situation is true even in the absence of a conservation law. For example, it can be shown that the set of non-detectable unitary operations form a symmetry group, $G$ [5]. In other words, $G$ is the symmetry group which describes the invariance of the system under the SSR. Moreover, this invariance implies that the set of physically allowed operations $\{\mathcal{O}(\cdot)\}$ is constrained to commute with the symmetry group $G[\underline{6}]$, that is,

$$
\mathcal{O}\left[\hat{T}(g) \hat{\rho} \hat{T}^{\dagger}(g)\right]=\hat{T}(g) \mathcal{O}[\hat{\rho}] \hat{T}^{\dagger}(g) \quad \forall g \in G
$$

for every state $\hat{\rho}$ of the system. Here $\hat{T}(g)$ is the unitary representation of the transformation $g \in G$. In general, $\mathcal{O}$ represents an operation on an open system and the openness implies that any global conservation law may not hold for the system itself. This shows that SSRs can exist independently of conservation laws at least for open systems.

In accord with previous work, we label the SSR associated with the symmetry group $G$ as the $G$-SSR. Alternatively, if $G$ is characterized by a generator, we sometimes refer to the SSR by the name of the generator. For example, a unitary representation of $\mathrm{U}(1)$ for a system of identical particles is given by $\left\{e^{i \hat{N} \theta}: 0 \leq \theta<2 \pi\right\}$ where the group generator $\hat{N}$ is the particle number operator, and so here the U(1)-SSR is equivalent to the particle number SSR.

The situation is further enriched if, in addition to a SSR, we also impose the constraint of allowing only local operations and classical communication (LOCC) for a bipartite system. The physically allowed operations $\mathcal{O}$ must satisfy both Eq. (1.1) and LOCC. Consider the bipartite case with subsystems at two spatially separated sites $\mathrm{A}$ and $\mathrm{B}$. Any local operation $\mathcal{O}=\mathcal{O}_{A} \otimes \mathbb{1}_{B}$ that 
satisfies Eq. (1.1) has the property that

$$
\left(\mathcal{O}_{A} \otimes \mathbb{1}_{B}\right)\left[\hat{T}(g) \hat{\rho} \hat{T}^{\dagger}(g)\right]=\hat{T}(g)\left(\mathcal{O}_{A} \otimes \mathbb{1}_{B}\right)[\hat{\rho}] \hat{T}^{\dagger}(g)
$$

for all $g \in G$, where $\mathcal{O}_{\mu}$ is a local operator acting at site $\mu$ and $\mathbb{1}$ is the identity operator. If the group has local representations $\left\{\hat{T}_{\mu}(g): g \in G\right\}$ for each site $\mu$ where $\hat{T}(g)=\hat{T}_{A}(g) \otimes \hat{T}_{B}(g)$ then

$$
\mathcal{O}_{\mu}\left[\hat{T}_{\mu}(g) \hat{\rho} \hat{T}_{\mu}^{\dagger}(g)\right]=\hat{T}_{\mu}(g) \mathcal{O}_{\mu}[\hat{\rho}] \hat{T}_{\mu}^{\dagger}(g) \quad \forall g \in G .
$$

For the case of a Lie group, this requires its local generators to be additive. Thus the combination of LOCC and a SSR can induce a local SSR acting at each site. Such local SSRs are not necessarily fundamental in the sense of arising from a conservation law like that of charge, but are imposed by our interest in examining the effect of the LOCC constraint. Essentially, a local SSR arises when two sites lack a shared reference to break the symmetry represented by $G$ at each site [ $5,6,6,6,6]$. However, the absence of the shared reference here is due to the LOCC constraint rather than any fundamental constraint. For example, the local U(1)-SSR due to the absence of a shared optical phase reference is imposed by the prohibition on transporting quantum fields between two sites under the LOCC constraint rather than the conservation of photon number.

Interest in superselection rules and their associated reference systems has revived in recent years particularly in relation to quantum information [9]. Bartlett et al. have shown that in the absence of a reference for spatial orientation the communication of classical and quantum information using spin particles is still possible provided the spins are entangled and the information is encoded in SSR-invariant subsystems [10]. They have also shown how a private shared reference for spatial orientation can be used for secret communications [11]. The effects of finite references has been studied in a variety of ways.

For example, Bartlett et al. [10], Bagan et al. [12] and Lindner et al. [13] have examined protocols for estimating the relative angle between two directions which are defined by finite dimensional spin systems. Further, the effect on quantum operations due to finite references was studied initially by van Enk and Kimble [14] and shortly later by Gea-Banacloche [15] and, although specific details of this initial work attracted some criticism from Itano [16] and Nha and Carmichael [17], it has been extended and generalized to quantum measurements, uncertainty relations and simultaneous measurements mainly by Ozawa [18, 19, 20, 21].

Entanglement is also affected by the presence of a SSR. A SSR has an effective decohering effect that constrains the amount of entanglement in a system that is accessible. A number of different terms have been used to label the entanglement which is constrained in this way. Bartlett and Wiseman [6], as well as others (see e.g. $[5,8,[22,[23])$, refer to it as the accessible entanglement [37]. For the special case of indistinguishable particles in the absence of a shared phase reference, we previously referred to the SSR-constrained entanglement as particle entanglement in Refs. [7, 8] to distinguish it from the entanglement of the spatial modes occupied by the particles. Our operational definition of particle entanglement makes use of a set of local quantum ancillary systems (or registers) which are not subject to the SSR. The accessible entanglement in a system of shared identical particles is given by the maximum amount of entanglement that one can transfer from the system to the local ancillas by $G$-invariant operations that obey LOCC. It is important to note that the transfer operation involves only a single copy of the system state. However, once the entanglement resides in the ancillae it can be manipulated in the usual way free of the SSR. Further details of this nonasymptotic interpretation of particle entanglement can be found in the Introduction of Ref. [5].

In this paper we focus on the problem of finding the optimal reference state that maximizes the accessible entanglement of a system in the presence of the local particle number SSR. Under such a SSR, the pure state describing a single particle which shared coherently between two sites is physically equivalent to an equal mixture of states representing a single particle at one site and a single particle at the other. In general, the SSR constrains the accessible entanglement due to the unobservabilty of the coherences between eigenkets of different local particle number. The accessible entanglement in this case is the weighted average of the entanglement found in each subspace of fixed local particle number. The entanglement lost because of the SSR can be recovered with a reference ancilla and it is this problem that we focus on here. In particular, we examine cases where the number of particles in the reference ancilla is not arbitrarily large and we explore how effectively the reference ancilla increases the accessible entanglement in a variety of situations. In Section [II we calculate the optimal reference state for maximizing the accessible entanglement from a system containing a single shared particle where the number of particles in the reference system is fixed. We compare the amount of entanglement made accessible by the optimal state with that due to various other states in section III and make steps towards finding optimal reference state for a general state of the system in section IV]

\section{OPTIMAL REFERENCE STATE FOR A SINGLE SHARED PARTICLE}

\section{A. Particle entanglement}

We begin by briefly recalling the definition of the particle entanglement (or equivalently, the accessible entanglement) as defined by Wiseman and Vaccaro [7]. As shown above, SSRs can be induced by the lack of a shared reference or a conservation law. The origin of the SSR is unimportant for the main results of this paper. However, as the conservation of particle number was used in Ref. [7], we assume the conservation also holds here. The obser- 
vation of coherences between states of different particle number requires operations that do not conserve particle number. The conservation of particle number therefore implies that phase coherences between subspaces of the Hilbert space corresponding to different numbers of particles are unobservable. This means that phase shifts generated by $\exp (-i \hat{N} \theta)$, where $\hat{N}$ is the particle number operator and $\theta$ a phase angle, are not detectable. The group of undetectable transformations is therefore the group $\mathrm{U}(1)=\{\exp (-i \hat{N} \theta): 0 \leq \theta<2 \pi\}$ of phase shift operators, which induces the particle number superselection rule U-SSR. In the case of bipartite systems, we consider the SSR which is induced by imposing the conservation of particle number at each spatial site. The corresponding group is $\mathrm{U}_{\mathrm{AB}}(1)=\mathrm{U}_{\mathrm{A}}(1) \otimes \mathrm{U}_{\mathrm{B}}(1)$ where $\mathrm{U}_{\mu}(1)$ is the group of operators that generate phase shifts at site $\mu$. We call this the local particle number superselection rule, i.e. the local U-SSR.

Consider the pure state $\left|\Psi^{(N)}\right\rangle_{\text {AB }}$ of a system comprising a fixed number of indistinguishable particles shared between two spatially separated sites labeled $A$ and $B$. We have included a superscript in the label of the state to indicate the total (fixed) number of particles the state represents. The effective state under the local particle number SSR is not $\left|\Psi^{(N)}\right\rangle_{\mathrm{AB}}$ but rather a mixed state given by [6]

$$
\begin{aligned}
\hat{\rho}^{(N)}= & \int_{2 \pi} \frac{d \theta_{A}}{2 \pi} \int_{2 \pi} \frac{d \theta_{B}}{2 \pi}\left[e^{-i\left(\hat{N}_{A} \theta_{A}+\hat{N}_{B} \theta_{B}\right)}\left|\Psi^{(N)}\right\rangle_{\mathrm{AB}}\right] \\
& \times\left[A B\left\langle\Psi^{(N)}\right| e^{i\left(\hat{N}_{A} \theta_{A}+\hat{N}_{B} \theta_{B}\right)}\right] \\
= & \sum_{n=0}^{N} \hat{\Pi}_{\mathrm{AB}}^{(n, N-n)}\left|\Psi^{(N)}\right\rangle_{\mathrm{AB}}\left\langle\Psi^{(N)}\right| \hat{\Pi}_{\mathrm{AB}}^{(n, N-n)}
\end{aligned}
$$

where $\hat{\Pi}_{\mathrm{AB}}^{(n, N-n)}$ is a operator which projects onto the subspace representing $n$ particles at site $\mathrm{A}$ and $(N-n)$ at B. The entanglement of $\hat{\rho}^{(N)}$ is, by definition, the particle entanglement of $\left|\Psi^{(N)}\right\rangle_{\mathrm{AB}}$. We note that the effective state, $\hat{\rho}^{(N)}$, is a mixture of a set of mutually orthogonal, entangled pure states $\hat{\Pi}_{\mathrm{AB}}^{(n, N-n)}\left|\Psi^{(N)}\right\rangle_{\mathrm{AB}}$. Its entanglement is thus the average of the entanglement of each member of the set. This means that the particle entanglement is given by [7]

$$
E_{\mathrm{P}}\left(\left|\Psi^{(N)}\right\rangle_{\mathrm{AB}}\right) \equiv \sum_{n} p_{n} E_{M}\left(\left|\Psi_{\mathrm{proj}}^{(n)}\right\rangle_{\mathrm{AB}}\right)
$$

where

$$
\begin{aligned}
\left|\Psi_{n}^{(N)}\right\rangle_{\mathrm{AB}} & =\frac{\hat{\Pi}_{\mathrm{AB}}^{(n, N-n)}\left|\Psi^{(N)}\right\rangle_{\mathrm{AB}}}{\sqrt{p_{n}}} \\
p_{n} & ={ }_{A B}\left\langle\Psi^{(N)}\left|\hat{\Pi}_{\mathrm{AB}}^{(n, N-n)}\right| \Psi^{(N)}\right\rangle_{A B} \\
E_{M}\left(\left|\Psi_{n}^{(N)}\right\rangle_{\mathrm{AB}}\right) & =S\left(\operatorname{Tr}_{A}\left[\left|\Psi_{n}^{(N)}\right\rangle_{\mathrm{AB}}\left\langle\Psi_{n}^{(N)}\right|\right]\right) .
\end{aligned}
$$

Here $S(\hat{\varrho})$ is the von Neumann entropy of $\hat{\varrho},\left|\Psi_{n}^{(N)}\right\rangle_{\mathrm{AB}}$ is the state of the system after detecting $n$ particles at site A, and $E_{M}(|\psi\rangle)$ is the entropy of entanglement of the state $|\psi\rangle$. In the current context $E_{M}(|\psi\rangle)$ corresponds to the entanglement of the spatial modes that are occupied by the particles.

\section{B. Particle entanglement of system and reference ancilla}

Two of us previously showed [7] that two copies of a shared single-particle system contained particle entanglement whereas (as noted above) each of the systems does not. Evidently one system behaves as a reference for the other. We further developed developed this concept in Ref. [5] where we showed that a shared particle ancilla can be used as a reference to increase the accessible the entanglement in another system. We now examine the problem of finding the state of the reference ancilla that yields the maximum value of the particle entanglement of the combined system and reference ancilla for a given state of the system. We confine our analysis to situations where the system and reference ancilla are in pure states to avoid unnecessary detail. Also in accord with our assumption that the particle number is conserved, we constrain the total number of particles in each of the system and the reference ancilla to be fixed [38]. Our focus will initially be on the simplest case, that of a single shared particle in the state:

$$
\left|\Psi^{(1)}\right\rangle_{\mathrm{AB}}=\frac{1}{\sqrt{2}}\left(|0,1\rangle_{\mathrm{AB}}+|1,0\rangle_{\mathrm{AB}}\right)
$$

where the ket $|n, m\rangle$ AB represents the occupation of spatial modes at the sites $\mathrm{A}$ and $\mathrm{B}$ by $n$ and $m$ identical particles, respectively, in second quantization notation. It is straightforward to show that this state possesses no particle entanglement, i.e. $E_{P}\left(\left|\Psi^{(1)}\right\rangle_{\mathrm{AB}}\right)=0$ [7, 8]. Let the reference ancilla consist of $M$ particles of the same type as the system and be prepared in the pure state

$$
\left|\Phi^{(M)}\right\rangle_{\mathrm{AB}}=\sum_{n=0}^{M} c_{n}|n, M-n\rangle_{\mathrm{AB}}
$$

for which the coefficients $c_{n}$ satisfy the normalization condition

$$
\left\langle\Phi^{(M)} \mid \Phi^{(M)}\right\rangle=\sum_{n=0}^{M}\left|c_{n}\right|^{2}=1
$$

but are otherwise undetermined. This ancilla state has been intentionally constructed to have no particle entanglement itself, i.e. $E_{P}\left(\left|\Phi^{(M)}\right\rangle_{\mathrm{AB}}\right)=0$. Any particle entanglement in the combined system and ancilla state, i.e. in

$$
\left|C^{(M+1)}\right\rangle_{\mathrm{AB}}=\left|\Psi^{(1)}\right\rangle_{\mathrm{AB}} \otimes\left|\Phi^{(M)}\right\rangle_{\mathrm{AB}}
$$

will therefore be due to the ancilla's ability to ameliorate the effects of the local U-SSR. 
Expanding $\left|C^{(M+1)}\right\rangle_{\mathrm{AB}}$ in terms of states with fixed local particle number yields

$$
\left|C^{(M+1)}\right\rangle=\sum_{n=0}^{M+1} \sqrt{p_{n}}\left|C_{n}^{(M+1)}\right\rangle
$$

where $p_{n}=\left\langle C^{(M+1)}\left|\hat{\Pi}_{A B}^{(n, M+1-n)}\right| C^{(M+1)}\right\rangle$ and

$$
\left|C_{n}^{(M+1)}\right\rangle=\frac{1}{\sqrt{p_{n}}} \hat{\Pi}_{A B}^{(M+1, n)}\left|C^{(M+1)}\right\rangle
$$

is a state of representing a total of $n$ particles at site $\mathrm{A}$ and $(M+1-n)$ at site B. For convenience, we omit the site labels $\mathrm{AB}$ here and below when they are implied by the context. The projection operator $\hat{\Pi}_{A B}^{(n, M+1-n)}$ is of the same basic form as the one in Eq. (2.4) but here it projects onto the subspace of states of the combined system plus ancilla with a total of exactly $n$ particles at $\mathrm{A}$ and $(M+1-n)$ at $\mathrm{B}$. That is, it distinguishes between the sites but it is insensitive to the individual modes at each site. Making use of Eq. (2.5) we find that

$$
\begin{array}{r}
\left|C_{n}^{(M+1)}\right\rangle=\frac{1}{\sqrt{2 p_{n}}}\left(c_{n-1}|1,0\rangle \otimes|n-1, M-n+1\rangle\right. \\
\left.+c_{n}|0,1\rangle \otimes|n, M-n\rangle\right)
\end{array}
$$

for $n=0,1, \ldots, M+1$. To make expressions more compact here and in the following, we have introduced two extra coefficients $c_{-1}$ and $c_{M+1}$ whose values are zero, i.e.

$$
\left|c_{-1}\right|^{2}=\left|c_{M+1}\right|^{2}=0 .
$$

We shall refer to Eq. (2.12) as the "boundary conditions". As before, the value of $p_{n}$ ensures that $\left\langle C_{n}^{(M+1)} \mid C_{n}^{(M+1)}\right\rangle=1$ and is easily found to be given by

$$
p_{n}=\frac{1}{2}\left(\left|c_{n-1}\right|^{2}+\left|c_{n}\right|^{2}\right)
$$

for $0 \leq n \leq M+1$. Taking the partial trace of $\left|C_{n}^{(M+1)}\right\rangle\left\langle C_{n}^{(\overline{M+1})}\right|$ over a basis representing states at site $\mathrm{B}$ yields

$$
\begin{aligned}
& \frac{\left|c_{n-1}\right|^{2}}{2 p_{n}}(|n-1\rangle \otimes|1\rangle\langle 1| \otimes\langle n-1|) \\
+ & \frac{\left|c_{n}\right|^{2}}{2 p_{n}}(|n\rangle \otimes|0\rangle\langle 0| \otimes\langle n|) .
\end{aligned}
$$

The entanglement of modes $E_{M}\left(\left|C_{n}^{(M+1)}\right\rangle\right)$ is given by the von Neumann entropy of Eq. (2.14), i.e.

$$
\begin{array}{r}
E_{\mathrm{M}}\left(\left|C_{n}^{(M+1)}\right\rangle\right)=- \\
\frac{1}{2 p_{n}}\left|c_{n-1}\right|^{2} \log _{2}\left(\frac{\left|c_{n-1}\right|^{2}}{2 p_{n}}\right) \\
-\frac{1}{2 p_{n}}\left|c_{n}\right|^{2} \log _{2}\left(\frac{\left|c_{n}\right|^{2}}{2 p_{n}}\right),
\end{array}
$$

and so, from Eq. (2.3), the particle entanglement is the average

$$
\begin{aligned}
E_{\mathrm{P}}\left(\left|C^{(M+1)}\right\rangle\right)=\sum_{n=0}^{M+1} p_{n} E_{\mathrm{M}}\left(\left|C_{n}^{(M+1)}\right\rangle\right) \\
=\frac{1}{2 \log _{2} e} \sum_{n=0}^{M+1}\left[-2\left|c_{n}\right|^{2} \ln \left(\left|c_{n}\right|^{2}\right)\right. \\
\left.\quad+\left(\left|c_{n-1}\right|^{2}+\left|c_{n}\right|^{2}\right) \ln \left(\left|c_{n-1}\right|^{2}+\left|c_{n}\right|^{2}\right)\right] .
\end{aligned}
$$

\section{Conditions for the optimum reference}

The maximization of the particle entanglement in Eq. (2.16) over the coefficients $c_{n}$ is subject to the normalization of the ancilla state $\left|\Phi^{(M)}\right\rangle$ given in Eq. (2.7). We note that the optimization can be performed with respect to $\left|c_{n}\right|^{2}$ rather than $c_{n}$ since the particle entanglement Eq. (2.16) and the constraint Eq. (2.7) are both functions of $\left|c_{n}\right|^{2}$ only. Let

$$
F=\left(2 \log _{2} e\right) E_{\mathrm{P}}\left(\left|C^{(M+1)}\right\rangle\right)-\alpha\left(\sum_{n=0}^{M}\left|c_{n}\right|^{2}-1\right)
$$

be the auxiliary function where $\alpha$ is a Lagrange multiplier.

The coefficients of the optimal reference state are given by the extremum of Eq. (2.17) and so satisfy

$$
\begin{aligned}
\frac{\partial F}{\partial\left|c_{n}\right|^{2}=}- & 2\left[\ln \left(\left|c_{n}\right|^{2}\right)+1\right]+\left[\ln \left(\left|c_{n-1}\right|^{2}+\left|c_{n}\right|^{2}\right)+1\right] \\
& +\left[\ln \left(\left|c_{n}\right|^{2}+\left|c_{n+1}\right|^{2}\right)+1\right]-\alpha \\
= & \ln \left[\frac{\left(\left|c_{n-1}\right|^{2}+\left|c_{n}\right|^{2}\right)\left(\left|c_{n}\right|^{2}+\left|c_{n+1}\right|^{2}\right)}{\left|c_{n}\right|^{4}}\right]-\alpha \\
= & 0
\end{aligned}
$$

for $n=0,1, \ldots, M$. This equation can be expressed in terms of a recurrence relation as follows

$$
\beta\left|c_{n}\right|^{4}=\left|c_{n}\right|^{4}+\left|c_{n-1}\right|^{2}\left|c_{n+1}\right|^{2}+\left|c_{n}\right|^{2}\left(\left|c_{n-1}\right|^{2}+\left|c_{n+1}\right|^{2}\right)
$$

for $n=0,1, \ldots, M$ where we have set $\beta=e^{\alpha}$. The boundary conditions in Eq. (2.12) ensure the recurrence relation has the correct form for $n=0$ and $n=M$.

To find the solution it is convenient to first rearrange the recurrence relation as

$$
\left|c_{n+1}\right|^{2}=\frac{(\beta-1)\left|c_{n}\right|^{4}-\left|c_{n}\right|^{2}\left|c_{n-1}\right|^{2}}{\left|c_{n}\right|^{2}+\left|c_{n-1}\right|^{2}} .
$$

Iterations of this starting from $n=0$ lead to explicit expressions for the coefficients in which $\left|c_{n}\right|^{2}$ is a polynomial in $\beta$ of order $n$. The details are given in the Appendix. Unfortunately, the derivation of a solution from these expressions that satisfies the upper boundary condition 


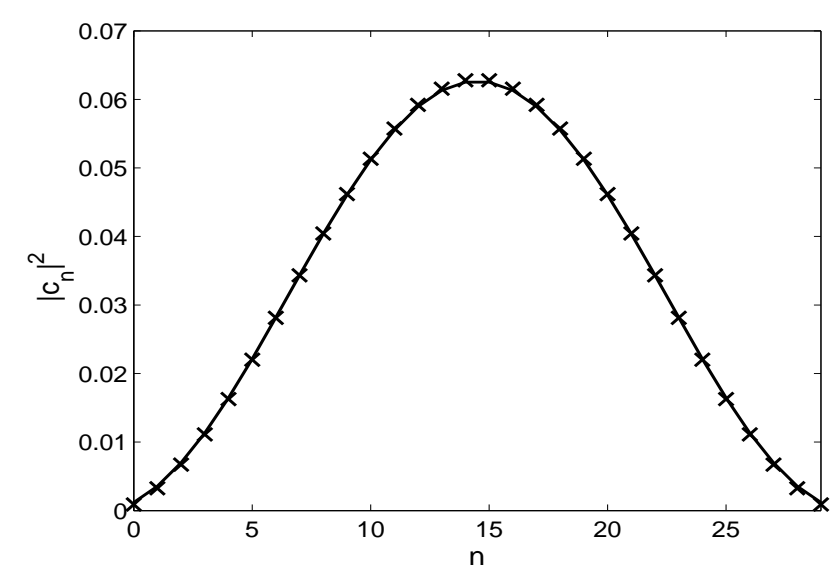

FIG. 1: Optimal reference state for a system comprising a single shared particle. The probability $\left|c_{n}\right|^{2}$ is plotted as a function of the number of particles $n$ at site A for the numerical solution (crosses) and our ansatz (solid curve) for an ancilla with a total of $M=29$. For the ansatz we used the values $A=1$ and $\epsilon=\frac{3}{2}$ which are correct to $O\left(M^{-2}\right)$.

$\left|c_{M+1}\right|^{2}=0$ and the normalization condition Eq. (2.7) does not appear tractable analytically. Nevertheless we note that the system state Eq. (2.5) is symmetric with respect to the interchange of labels, $|n, m\rangle_{\mathrm{AB}} \mapsto|m, n\rangle_{\mathrm{AB}}$, at each site $\mathrm{A}$ and $\mathrm{B}$, which implies that if there is a unique optimal reference state, it will also be symmetric under the same operation. Indeed we also show in the Appendix using the polynomial expressions that

$$
\left|c_{n}\right|^{2}=\left|c_{M-n}\right|^{2}
$$

for $n=0,1, \ldots, M$. This result will be useful later.

The optimal reference state for any given value of $M$ can be determined numerically by computing the values of $\beta$ and the set of coefficients $\left\{\left|c_{n}\right|^{2}\right\}$ that satisfy the recurrence relation Eq. (2.20), the normalization condition Eq. (2.7) and the boundary conditions Eq. (2.12). The approach we adopted was as follows: (i) make an initial guess of the value of $\beta$, (ii) use the lower boundary condition $\left|c_{-1}\right|^{2}=0$ and the recurrence relation Eq. (2.19) to compute the values of the variables $\left|c_{n}^{\prime}\right|^{2}=\left|c_{n}\right|^{2} /\left|c_{0}\right|^{2}$ for $n=0,1, \ldots, M$, (iii) use the computed values of $\left|c_{n}^{\prime}\right|^{2}$ and the normalization condition Eq. (2.7) to determine the values of the normalized coefficients $\left|c_{n}\right|^{2}$. This yields a set of normalized coefficients $\left|c_{0}\right|^{2},\left|c_{1}\right|^{2} \ldots,\left|c_{M}\right|^{2}$ that depend on the value of $\beta$. (iv) adjust the value of $\beta$ using a bisection method to find the zero of $\left|c_{M+1}\right|^{2}$ and repeat steps (ii)-(iv) until a desired tolerance is reached. In Fig. [1 we plot the numerically determined solution to the recurrence relation Eq. (2.19) for $M=29$. The figure clearly illustrates the predicted symmetry about $n=(M+1) / 2$.

In Fig. 22 we plot the particle entanglement, $E_{P}\left(\left|C^{(M+1)}\right\rangle\right)$, of the combined system plus ancilla against the total number of particles, $M$, in the ancilla.

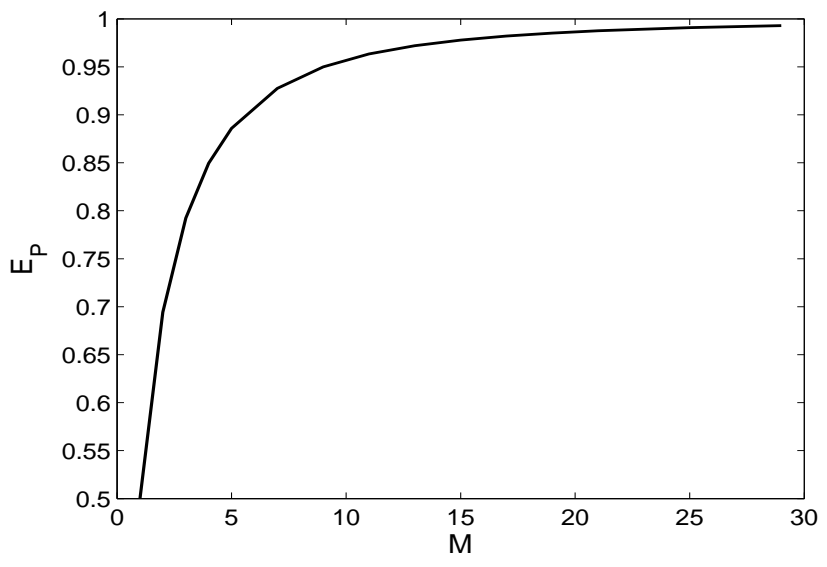

FIG. 2: The amount of particle entanglement $E_{P}$ versus the number of particles in the reference ancilla, $M$, for a system consisting of a single shared particle. The ancilla is in the optimal reference state discussed in the text.

The fact that $E_{P}\left(\left|C^{(M+1)}\right\rangle\right)>0$ whereas $E_{P}\left(\left|\Psi^{(1)}\right\rangle\right)=$ $E_{P}\left(\left|\Phi^{(M)}\right\rangle\right)=0$ shows that the ancilla partially shields the entanglement of the system from the local U-SSR.

\section{An ansatz for the optimal reference}

The numerical solution depicted in Fig. 1 1 suggests that the analytical solution may be of a trigonometric form. To check, we now consider the ansatz for the solution to the recurrence relation of the form

$$
\left|c_{n}\right|^{2}=B^{-1}\{A-\cos [z(n)]\}
$$

where

$$
z(n)=\xi n+\theta .
$$

Here $A, B, \xi$ and $\theta$ are constant for a given value of $M$, i.e. a given number of particles in the ancilla. We note that the numerical solution in Fig. (1) comprises almost one full cycle of the cosine function. This implies $\xi$ can be expressed conveniently in the form

$$
\xi=\frac{2 \pi}{M+2 \epsilon}
$$

where $\epsilon$ is an adjustable parameter. Moreover the symmetry condition Eq. (A8) implies a condition on the value of $\theta$ as follows. Replacing the left and right sides of Eq. (2.21) using the ansatz Eq. (2.22) and the expression for $\xi$ in Eq. (2.24) shows

$$
\cos \left[\frac{2 \pi}{M+2 \epsilon} n+\theta\right]=\cos \left[\frac{2 \pi}{M+2 \epsilon}(M-n)+\theta\right],
$$

which is satisfied by

$$
\theta=\frac{2 \pi \epsilon}{M+2 \epsilon}
$$


Hence the final form of our ansatz, given by Eqs. (2.22)(2.26) is

$$
\left|c_{n}\right|^{2}=B^{-1}\left\{A-\cos \left[\frac{2 \pi(n+\epsilon)}{M+2 \epsilon}\right]\right\}
$$

and depends on just three parameters $A, B$ and $\epsilon$.

We now check the validity of the ansatz by substituting it into the recurrence relation Eq. (2.19). To simplify matters we use the form given by Eqs. (2.22) and (2.23). The result of the substitution is

$$
\begin{aligned}
(\beta-1)\{ & A-\cos [z(n)]\}^{2} \\
=\{ & A-\cos [z(n+1)]\}\{A-\cos [z(n-1)]\} \\
& +\{A-\cos [z(n)]\} \\
& \times\{2 A-\cos [z(n+1)]-\cos [z(n-1)]\},
\end{aligned}
$$

which is independent of the normalization constant $B$. Using trigonometric identities and grouping terms it is easy to show that the right hand side of this equation can be expressed as

$$
\begin{aligned}
\mathrm{RHS}= & 3 A^{2}+\cos ^{2}(\xi)-1+[1+2 \cos (\xi)] \\
& \times\left\{-2 A \cos [z(n)]+\cos ^{2}[z(n)]\right\} .
\end{aligned}
$$

Similarly the left hand side of Eq. (2.22) is

$$
\text { LHS }=(\beta-1)\left\{A^{2}-2 A \cos [z(n)]+\cos ^{2}[z(n)]\right\} .
$$

Comparing the coefficients of $\cos [z(n)]$ and $\cos ^{2}[z(n)]$ in Eqs. (2.29) and (2.30) shows that the value of $\beta$ that satisfies the recurrence relation is given by

$$
\beta-1=2 \cos (\xi)+1 .
$$

Comparing the constant terms in these equations similarly shows that $(\beta-1) A^{2}=3 A^{2}-\sin ^{2}(\xi)$. These last two results imply that

$$
A^{2}=\frac{\sin ^{2}(\xi)}{2[1-\cos (\xi)]}=\frac{1+\cos (\xi)}{2} .
$$

Eliminating $\cos (\xi)$ and $\sin (\xi)$ in Eq. (2.32) using Eq. (2.31) then yields

$$
\beta=4 A^{2} .
$$

These results show that the ansatz does indeed satisfy the recurrence relation for particular values of the parameters $A, B$ and $\epsilon$.

To determine the actual values of the parameters we need to apply additional conditions. We first consider the boundary conditions $\left|c_{-1}\right|^{2}=0$ and $\left|c_{M+1}\right|^{2}=0$. Setting $n=-1$ in the detailed form of the ansatz Eq. (2.27) yields

$$
B^{-1}\left\{A-\cos \left[\frac{2 \pi(\epsilon-1)}{M+2 \epsilon}\right]\right\}=0,
$$

which gives an expression for $A$ in terms of $\epsilon$ as

$$
A=\cos \left[\frac{2 \pi(\epsilon-1)}{M+2 \epsilon}\right] .
$$

The same result is obtained for $n=M+1$ due to the symmetry condition Eq. (2.21). Finally we note that the analytic solution $\left|c_{1}\right|^{2}=\left|c_{0}\right|^{2}(\beta-1)$ given in Eq. (2.22) yields another expression involving $A$ and $\epsilon$ as follows. Using the ansatz Eq. (2.27) to replace the coefficients $\left|c_{0}\right|^{2}$ and $\left|c_{1}\right|^{2}$ and Eq. (2.33) to replace $\beta$ gives

$$
A-\cos \left[\frac{2 \pi(1+\epsilon)}{M+2 \epsilon}\right]=\left[A-\cos \left(\frac{2 \pi \epsilon}{M+2 \epsilon}\right)\right]\left(4 A^{2}-1\right) \text {. }
$$

This analysis shows that our ansatz, which depends on the values of just three parameters $A, B$ and $\epsilon$, satisfies all the conditions for the optimal reference state provided Eqs. (2.35) and (2.36) are satisfied. Indeed the values of the parameters $\epsilon$ and $A$ are determined by solving Eqs. (2.35) and (2.36) simultaneously, and the value of $B$ determined from the normalization condition Eq. (2.7). Once these values are determined, our ansatz Eq. (2.27) provides an exact analytical expression for the optimal reference state for any value of $M$.

We can also find approximate values for $A, B$ and $\epsilon$ in the large $M$ regime. Substituting for $A$ using Eq. (2.35) and simplifying yields

$$
\begin{aligned}
& \sin \left(\frac{2 \pi \epsilon}{M+2 \epsilon}\right) \sin \left(\frac{2 \pi}{M+2 \epsilon}\right) \\
& =\sin \left[\frac{2 \pi\left(\epsilon-\frac{1}{2}\right)}{M+2 \epsilon}\right] \sin \left(\frac{2 \pi \frac{1}{2}}{M+2 \epsilon}\right) \\
& \quad \times\left\{4 \cos ^{2}\left[\frac{2 \pi(\epsilon-1)}{M+2 \epsilon}\right]-1\right\}
\end{aligned}
$$

which, in the large $M$ regime, gives

$$
\epsilon=\frac{3}{2}+O\left(M^{-2}\right) .
$$

Similarly, we find from Eq. (2.35) in the same regime that

$$
A=1+O\left(M^{-2}\right) .
$$

The normalization constant $B$ is found by evaluating $\sum_{n}\left|c_{n}\right|^{2}=1$ using the ansatz Eq. (2.27), i.e.

$$
B=\sum_{n=0}^{M}\left\{A-\cos \left[\frac{2 \pi(n+\epsilon)}{M+2 \epsilon}\right]\right\} .
$$

For the case where $M$ is odd we find, using the symmetry condition Eq. (2.21), that

$$
B=2 \sum_{n=0}^{\frac{M-1}{2}} f(n)
$$

where

$$
f(x)=\left\{A-\cos \left[\frac{2 \pi(x+\epsilon)}{M+2 \epsilon}\right]\right\}
$$


is a monotonically increasing function over the range from $x=0$ to $x=\frac{M+1}{2}$. We note that

$$
2 \int_{0}^{\frac{M+1}{2}} d x f(x) \geq B \geq 2 \int_{0}^{\frac{M+1}{2}} d x f(x-1)
$$

that is,

$$
A(M+1)+1+\epsilon \geq B \geq A(M+1)-3+\epsilon,
$$

and so

$$
B^{-1}=\frac{1}{A(M+1)}+O\left(M^{-2}\right)
$$

The same result is also found for the case where $M$ is even. Hence in the large $M$ regime the analytical form of the optimal reference state is

$$
\begin{aligned}
\left|c_{n}\right|^{2} & =\frac{1}{M+1}\left\{1-\cos \left[\frac{2 \pi(n+3 / 2)}{M+3}\right]\right\}+O\left(M^{-2}\right) \\
& =\frac{2}{M+1} \sin ^{2}\left[\frac{\pi(n+3 / 2)}{M+3}\right]+O\left(M^{-2}\right)
\end{aligned}
$$

For comparison, in Fig. 1 we have also plotted as the solid curve the values of the probabilities $\left|c_{n}\right|^{2}$ given by the ansatz in Eq. (2.27) for $M=29$ for the values $\epsilon=\frac{3}{2}$ and $A=1$, which are correct to $O\left(M^{-2}\right)$. The figure shows that the ansatz with these approximate values of $\epsilon$ and $A$ is in relatively close agreement with the exact numerical solution (crosses) at this value of $M$.

\section{E. General nature of the optimal reference states}

To see why the optimal reference state has the general form shown in Fig. 1 and Eq. (2.45) consider two reference states in opposing limiting cases. First, in the limit of a narrow particle number distribution only one coefficient is non-zero; it is straightforward to show using Eq. (2.16) that the particle entanglement $E_{P}\left(\left|C^{(M+1)}\right\rangle\right)$ is zero for this reference state. In the other extreme, the particle number distribution is uniform with $\left|c_{n}\right|^{2}=$ $1 /(M+1)$ for $n=0,1, \ldots, M$; the right hand side of Eq. (2.16) is $1-M /(M+1)$ for this reference state. Clearly a reference system with broad particle number distribution has an advantage for making particle entanglement accessible. However, the reason for $E_{P}\left(\left|C^{(M+1)}\right\rangle\right)$ being less than 1 bit in the broad particle number distribution case can be traced to the boundary conditions Eq. (2.12). This suggests that a distribution which vanishes in near the boundaries $n=0$ and $n=M$ also has an advantage. Evidently the particle number distribution of the optimal reference state balances these two opposing effects, breadth of the distribution with vanishing values near the boundaries, to maximize $E_{P}\left(\left|C^{(M+1)}\right\rangle\right)$.

Our derivation of the optimal state fixes only the modulus of the coefficients $c_{n}$ in Eq. (2.6). The arguments of the complex numbers, $c_{n}$, are completely arbitrary. This is somewhat surprising given the role of the ancilla is presumed to act as a phase reference. We now explore how the ability to act as a reference for the U-SSR differs from the ability to act as a phase reference. First note that the asymmetry of a state depends solely on the modulus of number state coefficients $c_{n}[5]$. For example, consider the state of the reference ancilla representing a uniform sharing of particles with arbitrary complex arguments given by

$$
\left|\Phi^{(M)}\right\rangle_{\mathrm{AB}}=\frac{1}{\sqrt{M+1}} \sum_{n=0}^{M} e^{i \theta_{n}}|n, M-n\rangle_{\mathrm{AB}}
$$

where $\left\{\theta_{n}: 0 \leq \theta_{n}<2 \pi\right\}$ are an unordered set of phase angles. Under the local group operation $\hat{T}_{\mathrm{A}}(k \Delta)=$ $e^{-i \hat{N}_{\mathrm{A}} k \Delta} \otimes \mathbb{1}_{\mathrm{B}}$ where $\Delta=2 \pi /(N+1)$ this state transforms to

$$
\frac{1}{\sqrt{M+1}} \sum_{n=0}^{M} e^{i\left(\theta_{n}-n \phi_{k}\right)}|n, M-n\rangle_{\mathrm{AB}}
$$

which is orthogonal to $\left|\Phi^{(M)}\right\rangle_{\mathrm{AB}}$ for integer $k$ satisfying $1 \leq k \leq N$. This shows that $\left|\Phi^{(M)}\right\rangle_{\mathrm{AB}}$ is asymmetric with respect to $\mathrm{U}(1)$ and thus breaks the local $\mathrm{U}(1)$ symmetry [39]. As this result is independent of the values of the phase angles $\left\{\theta_{n}\right\}$ it confirms that only the moduli of the number state coefficients are important in terms of the U(1)-SSR. Moreover, Eq. 2.16) shows that the particle entanglement of a system consisting of one shared particle and an ancilla in reference state $\left|\Phi^{(M)}\right\rangle_{\mathrm{AB}}$ is independent of the phases, $\left\{\theta_{n}\right\}$, of the reference state.

Next consider the phase properties of the state $\left|\Phi^{(M)}\right\rangle_{\mathrm{AB}}$ for various choices of the set of phase angles $\left\{\theta_{n}\right\}$. For this we use the Pegg-Barnett phase formalism for physical states in the infinite- $s$ limit [24, 25, 26]. The joint phase probability density $P_{\Phi}\left(\theta_{\mathrm{A}}, \theta_{\mathrm{B}}\right)$ for phase angles $\theta_{\mathrm{A}}$ and $\theta_{\mathrm{B}}$ which describe the phase operators $\hat{\phi}_{\mathrm{A}}$ and $\hat{\phi}_{\mathrm{B}}$ of the ancilla spatial modes at sites $\mathrm{A}$ and $\mathrm{B}$, respectively, is given by

$$
P\left(\theta_{\mathrm{A}}, \theta_{\mathrm{B}}\right)=\frac{1}{(2 \pi)^{2}}\left|\sum_{n, m} e^{i\left(n \theta_{\mathrm{A}}+m \theta_{\mathrm{B}}\right)}\left\langle n, m \mid \Phi^{(M)}\right\rangle_{\mathrm{AB}}\right|^{2}
$$

For the reference state $\left|\Phi^{(M)}\right\rangle_{\mathrm{AB}}$ this can be rewritten as

$$
P\left(\theta_{\mathrm{A}}, \theta_{\mathrm{B}}\right)=\frac{1}{2 \pi} \mathcal{P}_{\Phi}\left(\theta_{\mathrm{A}}-\theta_{\mathrm{B}}\right)
$$

where

$$
\mathcal{P}_{\Phi}\left(\theta_{\mathrm{A}}-\theta_{\mathrm{B}}\right)=\frac{1}{2 \pi(M+1)}\left|\sum_{n=0}^{M} e^{i\left[n\left(\theta_{\mathrm{A}}-\theta_{\mathrm{B}}\right)+\theta_{n}\right]}\right|^{2}
$$

is the probability density for the phase difference $\hat{\phi}_{\mathrm{A}}-\hat{\phi}_{\mathrm{B}}$ and the factor $1 / 2 \pi$ in Eq. (2.48) is the uniform phase 


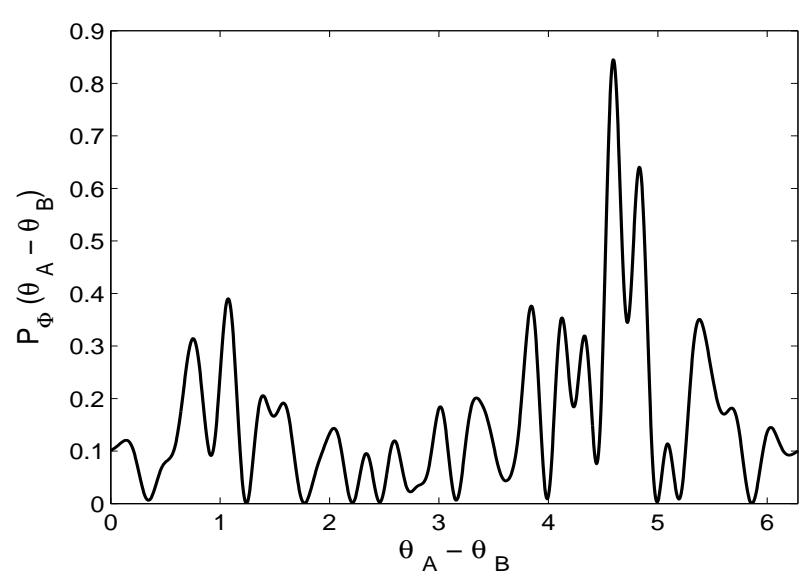

FIG. 3: The distribution of the relative phase difference for a random distribution of $\left\{\theta_{n}\right\}$.

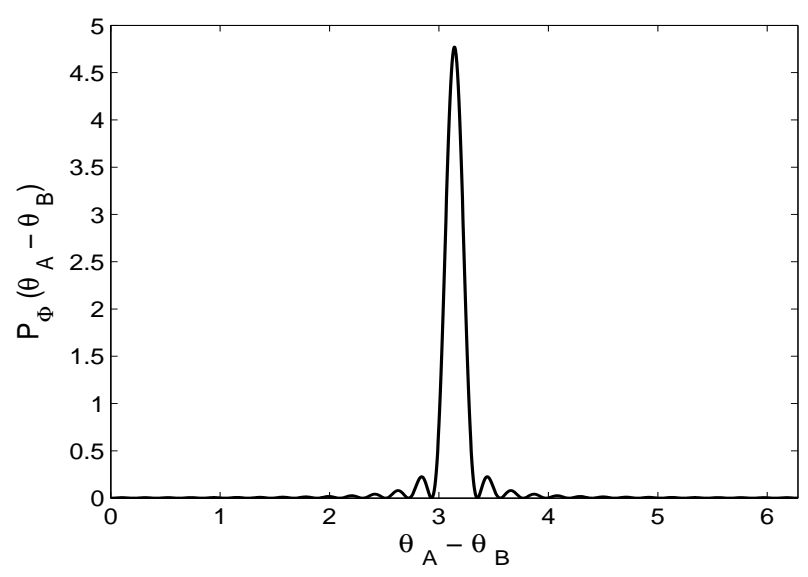

FIG. 4: The distribution of the relative phase difference where $\left\{\theta_{n}\right\}$ is linear in $n$.

probability density for the phase operator of either spatial mode. The highly correlated nature of the phase difference stems from the sharing of a fixed number $M$ of particles between the two modes. Figure 3 shows this phase distribution for the case where $\theta_{n}=\pi n$ whereas figure 4 shows the phase distribution where $\theta_{n}$ is random. Note that both phase distributions equally alleviate the U-SSR. Clearly there is a fundamental difference between the ability to act as a phase reference and the ability to act as a reference for the U-SSR, viz. the complex phase of the number state coefficients are crucial for the former but are unimportant for the latter. While this conclusion applies to the specific case of a single-shared particle system as considered in Eq. (2.16) , we show later in Section IV that it also extends to arbitrary systems states.

\section{COMPARISON OF THE OPTIMAL REFERENCE FRAME TO OTHER STATES}

States with broad particle number distributions are known to have well-defined phase properties 24, 25, 26, 27]. So it is interesting to compare our optimal reference states with classes of states that have been optimized for other phase dependent quantities. We define a figure of merit, $D$, based on the excess particle entanglement that is made accessible by the optimal reference state as follows:

$$
D=\frac{E_{\mathrm{p}}\left[\left|C_{\mathrm{opt}}^{(M+1)}\right\rangle\right]-E_{\mathrm{p}}\left[\left|C^{(M+1)}\right\rangle\right]}{E_{\mathrm{p}}\left[\left|C_{\mathrm{opt}}^{(M+1)}\right\rangle\right]},
$$

where

$$
\begin{aligned}
& \left|C_{\mathrm{opt}}^{(M+1)}\right\rangle=\left|\Psi^{(1)}\right\rangle \otimes\left|\Phi_{\mathrm{opt}}^{(M)}\right\rangle \\
& \left|C^{(M+1)}\right\rangle=\left|\Psi^{(1)}\right\rangle \otimes\left|\psi^{(M)}\right\rangle,
\end{aligned}
$$

for single particle system state $\left|\Psi^{(1)}\right\rangle$, optimal reference ancilla state $\left|\Phi_{\mathrm{opt}}^{(M)}\right\rangle$ and arbitrary reference ancilla state $\left|\psi^{(M)}\right\rangle$. We now consider a number of states whose phase properties are important in some way. All the states are constructed on the Hilbert space $\mathbb{H}_{\mathrm{R}}$ which is spanned by the number state basis $\{|n, M-n\rangle: n=0,1, \ldots, M\}$.

The Berry-Wiseman phase optimized states of a twomode optical field give the optimum phase shift estimation in a Mach-Zehnder apparatus for a fixed total number of particles (photons) under ideal canonical phase measurements [28]. These states have the form

$$
|\psi\rangle \propto \sum_{n=0}^{M} \sin \left[\frac{\pi(n+\epsilon)}{M+2 \epsilon}\right]|n, M-n\rangle
$$

where $M$ is the number of particles and $\epsilon=1$. The Summy-Pegg phase optimized states of a single mode optical field give the minimum phase variance for a fixed upper bound in the particle (photon) number distribution 27]. Their two-mode version on the Hilbert space $\mathbb{H}_{R}$ is given by Eq. (3.4) with a parameter value of $\epsilon \approx 0.84$ for $M \gtrsim 10$. The variance of the optimized phase quantity for both classes of states scales as $1 / M^{2}$. A class of states with less phase resolution is given by the single-mode coherent states $|\alpha\rangle \propto \sum_{n} \alpha^{n}|n\rangle / \sqrt{n !}$ where $|n\rangle$ is the usual number state. These states have a phase variance which scales as $1 /|\alpha|^{2}$ and are optimized in the sense that they approximate number-phase minimum uncertainty states [25] for $|\alpha| \gg 1$. To make a comparison with our optimal reference state we construct a two-mode version in the Hilbert space $\mathbb{H}_{R}$ with an analogous number state expansion to $|\alpha\rangle$ as follows

$$
|\phi\rangle \propto \sum_{n=0}^{M} \sqrt{\frac{\left(\frac{M}{2}\right)^{n}}{n !}}|n, M-n\rangle .
$$


The parameter that is analogous to the field amplitude $\alpha$ in $|\alpha\rangle$ has the value $\sqrt{M / 2}$ here. Thus in the large $M$ regime the variance of the two-mode phase operator scales as $2 / M$. We also consider reference states whose particle number probability distribution $\left|c_{n}\right|^{2}$ corresponds to a binomial distribution, i.e.

$$
|\psi\rangle=\sum_{n=0}^{M}\left[\left(\begin{array}{c}
M \\
n
\end{array}\right) p^{n}(1-p)^{(M-n)}\right]^{1 / 2}|n, M-n\rangle
$$

for which we set $p=0.5$ to make the distribution symmetric. These states are a two-mode generalization of Stoler et al.'s single mode binomial states [29]. Finally we include in our comparison the shared phase state,

$$
|\psi\rangle=\frac{1}{\sqrt{M+1}} \sum_{n=0}^{M}|n, M-n\rangle,
$$

which is a two mode version of the single-mode PeggBarnett phase state with zero phase [24, 26]. This state belongs to the class of states on $\mathbb{H}_{\mathrm{R}}$ with maximum asymmetry with respect to the particle number SSR [5]. The change in the von Neumann entropy of these states due to the action of the SSR is the maximum value of $\log _{2} M$ and so these states play an important role in terms of breaking the symmetry represented by the SSR [5].

Fig. 3 shows that the figure of merit, $D$, of all states, at least after a certain value of $M$, are monotonically decreasing with $M$. However, the decrease in $D$ for increasing $M$ for the Berry-Wiseman and the two-mode SummyPegg minimized phase variance states is far greater than the two-mode versions of the coherent and binomial states. The reason for this can be traced to our earlier observation in Sec. IIE that broad particle-number distributions are an advantage in making the entanglement accessible. In particular the former two states have a particle-number standard deviation that scales as $M$ whereas the two-mode coherent states have a particlenumber standard deviation that scales as $\sqrt{M}$. Compared to the maximum possible width $M$ of the distribution, the former are relatively broad and the latter is relatively narrow. We note that the figure of merit $D$ for the coherent and binomial states become closer as $M$ increases as expected from the fact that the associated particle-number distributions approach each other as $M \rightarrow \infty$.

\section{OPTIMAL REFERENCE STATES FOR GENERAL PURE STATES}

We now generalize our results to the case where there are $N$ particles in the system and, as before, $M$ particles in the ancilla. Consider the tensor product of two arbitrary states of the system and ancilla for this case given by

$$
\left|C^{(M+N)}\right\rangle_{\mathrm{AB}}=\left|\Psi^{(N)}\right\rangle_{\mathrm{AB}} \otimes\left|\Phi^{(M)}\right\rangle_{\mathrm{AB}}
$$

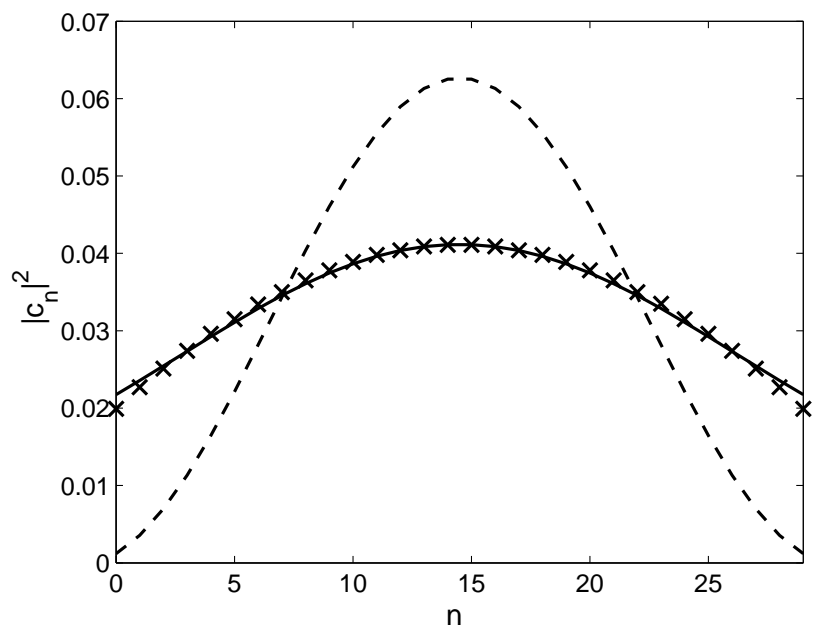

FIG. 5: A comparison of the figure of merit $D$ for the shared phase states (o), two-mode versions of the coherent states $(*)$, binomial states $(+)$ and Summy-Pegg phase optimized states $(\times)$, and Berry-Wiseman phase optimized states $(\square)$. $D$ represents the relative effectiveness of a given reference state compared to the optimal reference state. The effectiveness of all states increases as the total number of particles $M$ increases for large $M$.

where the system and ancilla states are

$$
\begin{aligned}
\left|\Psi^{(N)}\right\rangle_{\mathrm{AB}} & =\sum_{n=0}^{N} d_{n}|n, N-n\rangle_{\mathrm{AB}}, \\
\left|\Phi^{(M)}\right\rangle_{\mathrm{AB}} & =\sum_{n=0}^{M} c_{n}|n, M-n\rangle_{\mathrm{AB}},
\end{aligned}
$$

respectively. The coefficients $c_{n}$ and $d_{n}$ are subject to the normalization conditions

$$
\sum_{n=0}^{N}\left|d_{n}\right|^{2}=\sum_{m=0}^{M}\left|c_{m}\right|^{2}=1
$$

Neither the system nor the ancilla contain entanglement of particles i.e. $E_{\mathrm{P}}\left(\left|\Psi^{(M)}\right\rangle_{\mathrm{AB}}\right)=E_{\mathrm{P}}\left(\left|\Phi^{(M)}\right\rangle_{\mathrm{AB}}\right)=0$. To simplify the notation we shall omit the subscripts $A$ and $B$ from here onwards. The state of the combined system can be expressed as

$$
\begin{aligned}
\left|C^{M+N}\right\rangle & =\sum_{n=0}^{N} \sum_{m=0}^{M} d_{n} c_{m}|n, N-n\rangle \otimes|m, M-m\rangle \\
& =\sum_{k=0}^{N+M} \sqrt{p_{k}}\left|C_{k}^{(M+N)}\right\rangle
\end{aligned}
$$

where $\left|C_{k}^{(M+N)}\right\rangle$ is a state containing exactly $k$ particles at site $\mathrm{A}$ and $(N+M-k)$ at site $\mathrm{B}$. Also $p_{n}$ is a nor- 
malization constant, i.e.

$$
\begin{aligned}
\left|C_{k}^{(M+N)}\right\rangle= & \frac{1}{\sqrt{p_{k}}} \sum_{n=0}^{N} \sum_{m=0}^{M} \delta_{n+m, k} d_{n} c_{m} \\
& \times|n, N-n\rangle \otimes|m, M-m\rangle \\
p_{k}= & \sum_{n=0}^{N} \sum_{m=0}^{M}\left|d_{n} c_{m}\right|^{2} \delta_{n+m, k} .
\end{aligned}
$$

The calculation of $E_{\mathrm{P}}$ in Section $\amalg$ for a system comprising one particle is easily extended to the case $N$ particles as follows. We find that

$$
\begin{aligned}
& E_{\mathrm{P}}\left(\left|C^{(M+N)}\right\rangle\right)=\sum_{k=0}^{N+M} p_{k} E_{\mathrm{M}}\left(\left|C_{k}^{(M+N)}\right\rangle\right) \\
& =-\sum_{k=0}^{N+M} p_{k} \sum_{n=0}^{N} \sum_{m=0}^{M} \frac{\left|d_{n} c_{m}\right|^{2}}{p_{k}} \delta_{n+m, k} \log _{2} \frac{\left|d_{n} c_{m}\right|^{2}}{p_{k}} \\
& =-\sum_{k=0}^{N+M} \sum_{n=0}^{N} \sum_{m=0}^{M}\left|d_{n} c_{m}\right|^{2} \delta_{n+m, k} \log _{2} \frac{\left|d_{n} c_{m}\right|^{2}}{p_{k}}
\end{aligned}
$$

We again find that the particle entanglement is independent of the complex phases of the number state coefficients $c_{n}$ of the reference state. This shows that our conclusion in Section $\amalg \mathrm{E}$ for the special case of a single shared-particle system state, namely that a phase reference and a reference for the U-SSR are fundamentally different, applies also to the general case of arbitrary system states.

\section{A. The infinite-particle ancilla state}

One may suspect that in the limit of $M \rightarrow \infty$, the ancilla could resemble a classical reference and completely shield the system from the local particle number SSR. We now show this using the uniformly distributed ancilla state with $c_{n}=1 / \sqrt{M+1}$ for $M \gg N$. The state with exactly $k$ particles at site A for $N \leq k \leq M$ is given by

$$
\begin{aligned}
& \left|C_{k}^{(M+N)}\right\rangle \\
& \quad=\frac{1}{\sqrt{p_{k}}} \sum_{n=0}^{N} d_{n}|n, N-n\rangle \otimes|(k-n), M-(k-n)\rangle
\end{aligned}
$$

and so

$$
E_{\mathrm{P}}\left(\left|C_{k}^{(M+N)}\right\rangle\right)=E_{\mathrm{M}}\left(\left|C_{k}^{(M+N)}\right\rangle\right),
$$

and the probability $p_{k}$ in Eq. (4.6) of finding this state is simply

$$
p_{k}=\sum_{n=0}^{N} \frac{\left|d_{n}\right|^{2}}{M+1}=\frac{1}{M+1} .
$$

The contribution to $E_{\mathrm{P}}\left(\left|C^{(M+N)}\right\rangle\right)$ from these states is

$$
X=\sum_{k=N}^{M} p_{k} E_{\mathrm{M}}\left(\left|C_{k}^{(M+N)}\right\rangle\right)=\frac{M+1-N}{M+1} E_{\mathrm{M}}\left(\left|\Psi^{(N)}\right\rangle\right) .
$$

It is straightforward to show that the remaining $2 N$ states $\left|C_{k}^{(M+N)}\right\rangle$ for $0 \leq k<N$ and $M<k \leq N+M$ have $E_{\mathrm{M}}\left(\left|C_{k}^{(M+N)}\right\rangle\right) \leq E_{\mathrm{M}}\left(\left|\Psi^{(N)}\right\rangle\right)$ and $p_{k} \leq 1 /(M+1)$ and so their contribution to $E_{\mathrm{P}}\left(\left|C^{(M+N)}\right\rangle\right)$ is less than

$$
Y=\frac{2 N}{M+1} E_{\mathrm{M}}\left(\left|\Psi^{(N)}\right\rangle\right) .
$$

These last two results give bounds on $E_{\mathrm{P}}\left(\left|C^{(M+N)}\right\rangle\right)$ as

$$
X \leq E_{\mathrm{P}}(|C\rangle) \leq X+Y .
$$

In the limit $M \rightarrow \infty, X \rightarrow E_{\mathrm{M}}\left(\left|\Psi^{(N)}\right\rangle\right)$ and $Y \rightarrow 0$ and so

$$
E_{\mathrm{P}}\left(\left|C^{(M+N)}\right\rangle\right) \rightarrow E_{\mathrm{M}}\left(\left|\Psi^{(N)}\right\rangle\right) .
$$

The infinite-particle ancilla effectively shields the system from the particle number SSR and makes all of its entanglement accessible. We note that the class of perfect reference ancillae for the SSR include states that are poor phase references.

\section{B. Conditions for general optimal ancilla states}

We now use the Lagrange multiplier method to find conditions for the coefficients $c_{n}$ of the optimal ancilla state for an arbitrary, but fixed, value of $M$. We want to maximize the particle entanglement $E_{\mathrm{P}}\left(\left|C^{(M+N)}\right\rangle\right)$ over $\left|c_{n}\right|^{2}$ subject to the normalization constraint $\sum_{n=0}^{M}\left|c_{n}\right|^{2}=1$. The optimal ancilla state for any system state is given by the extremum of the auxiliary function

$$
F=E_{\mathrm{P}}\left(\left|C^{(M+N)}\right\rangle\right)-\alpha_{1}\left(\sum_{n=0}^{M}\left|c_{n}\right|^{2}-1\right)
$$

where $E_{\mathrm{P}}\left(\left|C^{(M+N)}\right\rangle\right)$ is given by Eq. (4.7) and $\alpha_{1}$ is the Lagrange multiplier. Setting the derivative $\partial F / \partial\left|c_{m}\right|^{2}$ to zero we find with a little effort that

$$
\alpha_{1}=\sum_{n=0}^{N}\left[-\left|d_{n}\right|^{2} \log _{2}\left(\left|d_{n} c_{m}\right|^{2}\right)+\left|d_{n}\right|^{2} \log _{2} p_{n+m}\right]
$$

for $m=0,1, \ldots M$ and where $p_{k}$ is given by Eq. (4.6).

It is easy to show that the symmetry condition Eq. (2.21) does not hold in general. Consider, for example, the simplest case given by $N=M=1$, where both the system and ancilla contain a single shared particle. Substituting these values into Eq. (4.11) yields for $m=0$

$$
\alpha_{1}=\left|c_{1}\right|^{2} \log _{2} \frac{\left|c_{1} d_{0}\right|^{2}+\left|c_{0} d_{1}\right|^{2}}{\left|c_{0} d_{0}\right|^{2}}
$$


and for $m=1$

$$
\alpha_{1}=\left|c_{0}\right|^{2} \log _{2} \frac{\left|c_{1} d_{0}\right|^{2}+\left|c_{0} d_{1}\right|^{2}}{\left|c_{0} d_{1}\right|^{2}} .
$$

The simultaneous solution of these equations is given by

$$
\frac{2^{x}-1}{x}=\left|\frac{d_{0}}{d_{1}}\right|^{2}
$$

where $x=\left|c_{1} / c_{0}\right|^{2}$. This implies that the optimum reference state for a non-symmetric system state, $\left|c_{0}\right| \neq\left|c_{1}\right|$, is also non-symmetric with $\left|d_{0}\right| \neq\left|d_{1}\right|$.

The analytical treatment of system and ancilla states with arbitrary values of $N$ and $M$, respectively, is beyond the scope the current paper. Instead we focus here on the situation where the system state is of a simple form, namely, a shared phase state with $d_{n}=1 / \sqrt{N+1}$, i.e. we set

$$
\left|\Psi^{(N)}\right\rangle=\frac{1}{\sqrt{N+1}} \sum_{n=0}^{N}|n, N-n\rangle .
$$

We further restrain the problem to the case where $N=$ $M$ to make the problem tractable.

A shared phase state has a symmetry between the sites in the sense that it is invariant under an interchange or site labels, i.e. $A B \mapsto B A$. The corresponding optimal reference state has the same symmetry with coefficients of the form

$$
\left|c_{m}\right|^{2}=\left|c_{M-m}\right|^{2}
$$

for $m=0,1, \ldots, M$. The proof of this is as follows. We find from Eqs. (4.11) and (4.6) the set of equations

$$
\alpha_{1}=-\log _{2}\left(\frac{1}{N+1}\left|c_{m}\right|^{2}\right)+\frac{1}{N+1} \sum_{n=0}^{N} \log _{2} p_{n+m}
$$

for $m=0,1, \ldots, M$ where

$$
p_{k}=\frac{1}{N+1} \sum_{j=k-N}^{k}\left|c_{j}\right|^{2} .
$$

Here, for compactness, we have extended the set of coefficients $c_{n}$ with the values $c_{n}=0$ for $n<0$ and $n>M$. Taking the exponential of both sides of Eq. (4.14) yields

$$
\beta\left|c_{m}\right|^{2(N+1)}=\prod_{n=0}^{N}\left(\sum_{j=n+m-N}^{n+m}\left|c_{j}\right|^{2}\right)
$$

Omitting the zero terms $\left|c_{j}\right|^{2}$ for $j<0$ and $j>M$ and setting $N=M$ we find

$$
\begin{aligned}
\beta\left|c_{m}\right|^{2(M+1)} & =\prod_{n=0}^{M-m}\left(\sum_{j=0}^{n+m}\left|c_{j}\right|^{2}\right) \prod_{n=M-m}^{M}\left(\sum_{j=n+m-M}^{M}\left|c_{j}\right|^{2}\right) \\
& =\prod_{n=m}^{M}\left(\sum_{j=0}^{n}\left|c_{j}\right|^{2}\right) \prod_{n=0}^{m}\left(\sum_{j=n}^{M}\left|c_{j}\right|^{2}\right)
\end{aligned}
$$

where we have made use of the normalization Eq. (4.3) to include an extra factor in the first line. Using this result we find the relationship between the coefficients $\left|c_{m}\right|^{2}$ for sequential values of the index $m$ is

$$
\begin{aligned}
& \beta\left|c_{m+1}\right|^{2(M+1)}=\beta\left|c_{m}\right|^{2(M+1)} \frac{1-\sum_{j=0}^{m}\left|c_{j}\right|^{2}}{\sum_{j=0}^{m}\left|c_{j}\right|^{2}} \\
& \beta\left|c_{m-1}\right|^{2(M+1)}=\beta\left|c_{m}\right|^{2(M+1)} \frac{1-\sum_{j=m}^{M}\left|c_{j}\right|^{2}}{\sum_{j=m}^{M}\left|c_{j}\right|^{2}} .
\end{aligned}
$$

We also find by setting specific values of $m$ in Eq. (4.17) that

$$
\begin{aligned}
\beta\left|c_{0}\right|^{2(M+1)} & =\prod_{n=0}^{M}\left(\sum_{j=0}^{n}\left|c_{j}\right|^{2}\right) \\
\beta\left|c_{M}\right|^{2(M+1)} & =\left(\sum_{j=0}^{M}\left|c_{j}\right|^{2}\right) \prod_{n=0}^{M-1}\left(\sum_{j=n}^{M}\left|c_{j}\right|^{2}\right)
\end{aligned}
$$

and so $\left|c_{0}\right|^{2}=\left|c_{M}\right|^{2}$. Using this result together with Eqs. (4.18) and (4.19) with the specific values of $m=0$ and $m=M$, respectively, shows that $\left|c_{1}\right|^{2}=\left|c_{M-1}\right|^{2}$. Repeating this analysis for sequential values of $m$ completes the proof of the symmetry in Eq. (4.13).

Another property of the optimal state is that the distribution $\left|c_{m}\right|^{2}$ is unimodal which can be seen as follows. First we define the difference $\Delta_{m+1, m}$ as

$$
\Delta_{m+1, m}=\left|c_{m+1}\right|^{2(M+1)}-\left|c_{m}\right|^{2(M+1)}
$$

which, from Eq. (4.18), is given by

$$
\Delta_{m+1, m}=\left|c_{m}\right|^{2(M+1)}\left(\frac{1-2 \sum_{j=0}^{m}\left|c_{j}\right|^{2}}{\sum_{j=0}^{m}\left|c_{j}\right|^{2}}\right) \text {. }
$$

From the symmetry property Eq. (4.13) of the optimal state and the normalization condition Eq. (4.3) we know that

$$
\sum_{m=0}^{[M / 2]}\left|c_{m}\right|^{2}=\sum_{m=[M / 2]+1}^{M}\left|c_{n}\right|^{2}=\frac{1}{2}
$$

where $[M / 2]$ is the largest integer satisfying $[M / 2] \leq$ $M / 2$. Next we use this result to determine the positivity of the differences $\Delta_{m+1, m}$. Consider if the expression $1-2 \sum_{j=0}^{m}\left|c_{j}\right|^{2}$ in Eq. (4.23) were zero for a value of $m$ less than $[M / 2]$. This would imply that $\left|c_{m+1}\right|^{2},\left|c_{m+2}\right|^{2}, \ldots,\left|c_{[M / 2]}\right|^{2}=0$ and by Eq. (4.23) that $\Delta_{m+1, m}=0$, which would mean that $\left|c_{m}\right|^{2}=\left|c_{m+1}\right|^{2}=$ 0 and so $1-2 \sum_{j=0}^{m-1}\left|c_{j}\right|^{2}=0$. It follows, by induction, that the coefficients $\left|c_{j}\right|^{2}$ would be zero for $j=0,1, \ldots,[M / 2]$ which contradicts Eq. (4.24). Hence $1-2 \sum_{j=0}^{m}\left|c_{j}\right|^{2}=0$ only for $m=[M / 2]$ and so from 


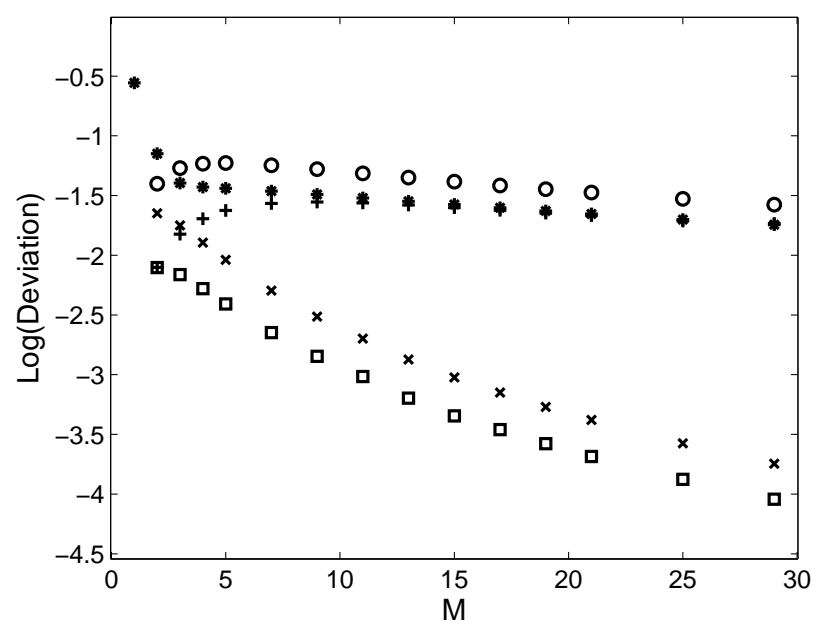

FIG. 6: Comparison of the particle number distributions for the optimal reference state $\left|\Phi_{\mathrm{opt}}^{(M)}\right\rangle(\times)$ and the trial state $\left|\phi_{\text {trial }}^{(M)}\right\rangle(-)$ for $N=M=29$. The comparison shows that the optimal reference state is very well approximated by the trial state. Moreover, a comparison with the optimal state for $N=1, M=29(--)$ shows that the optimal particle number distribution broadens out as the number of particles in the system, $N$, increases. Finally we note that distribution for the optimal reference state $(x)$ satisfies the symmetry and unimodal properties derived analytically.

Eq. (4.23) $\Delta_{j+1, j}>0$ for $j=0,1, \ldots,([M / 2]-1)$. Taking account of the symmetry Eq. (4.13) then shows that the distribution $\left|c_{n}\right|^{2}$ is monotonically increasing over the index $m$ for $m=0,1, \ldots,[M / 2]$ and monotonically decreasing for $m=[M / 2],([M / 2]+1), \ldots, M$. This completes the proof of the unimodal property.

In summary, the optimal state for a system in the shared phase state Eq. (4.12) and a reference ancilla with an equal maximum number of particles, i.e. with $M=N$, has coefficients $\left|c_{m}\right|$ that are symmetric about a modal point and are monotonically increasing before this point and are monotonically decreasing after it. These properties are reminiscent of the ansatz used for a single shared particle case which suggests that the optimal state would be well approximated by a trial state $\left|\phi_{\text {trial }}^{(M)}\right\rangle$ which is of the form Eq. (2.27) for a suitable choice of the parameters $A$ and $\epsilon$, and a range of $M$ values. Exact analytical solutions or better approximations are beyond the scope of this paper. However, one can solve the $N=M$ case numerically using either Eq. (4.18) or Eq. (4.19). Fig. 6 shows the particle number distribution for the exact optimal reference state with $N=M=29$ compared with that of the trial state for the parameter values $A=1.9$ and $\epsilon=8.9$. The figure confirms our intuition that the exact solution in the $N=M$ case can be approximated by a state which is of the form Eq. (2.27). The inner product $\left\langle\Phi_{\mathrm{opt}}^{(M)} \mid \phi_{\text {trial }}^{(M)}\right\rangle$ of the exact solution with the trial state differs from one by $6 \times 10^{-6}$.

\section{DISCUSSION}

We have focused on the situation where the ancilla state $\left|\Phi^{(M)}\right\rangle$ has a particularly simple form, as given by Eq. (2.6) and Eq. (4.2), corresponding to a single spatial mode at each site. Such states automatically satisfy the requirement $E_{\mathrm{P}}\left(\left|\Phi^{(M)}\right\rangle\right)=0$, i.e. that the ancilla does not contain particle entanglement. Nevertheless our analysis generalizes quite easily to situations with multiple spatial modes and multiple ancillae. The most general pure ancilla state of $M$ particles which contains no particle entanglement has the form

$$
\left|\Phi^{(M)}\right\rangle=\sum_{m=0}^{M} c_{m}|\phi(m)\rangle_{\mathrm{A}} \otimes|\varphi(M-m)\rangle_{\mathrm{B}}
$$

where $|\chi(n)\rangle_{\mu}$, for $\chi=\phi$ or $\varphi$, is an arbitrary normalized state of exactly $n$ particles at site $\mu$ in the second quantization formalism. For example, if the part of the ancilla at site $\mu$ involves the 3 mutually orthogonal spatial modes $|\cdot\rangle_{\mu} \otimes|\cdot\rangle_{\mu} \otimes|\cdot\rangle_{\mu}$, then the general expression for $|\chi(n)\rangle_{\mu}$ is

$$
|\chi(n)\rangle_{\mu}=\sum_{i, j, k} f_{i, j, k}|i\rangle_{\mathrm{A}} \otimes|j\rangle_{\mathrm{A}} \otimes|k\rangle_{\mathrm{A}} \delta_{i+j+k, n}
$$

where $f_{i, j, k}$ are normalized coefficients. Notice that the set of states at each site are orthonormal, viz. ${ }_{\mu}\langle\chi(n) \mid \chi(m)\rangle_{\mu}=\delta_{n, m}$. To incorporate these more general ancilla states in our previous analysis we need only replace the ancilla states $|n\rangle_{\mu}$ in the above with $\left|\phi_{\mu}(n)\right\rangle_{\mu}$. However, as our analysis used only the orthogonality property of the set of $|n\rangle_{\mu}$ and not its detailed structure, the coefficients $\left|c_{m}\right|^{2}$ for the optimal ancilla state would be unchanged. Hence the solutions derived above represent classes of optimal ancilla states for arbitrary choices of coefficients of the kind $f_{i, j, k}$ in Eq. (5.2).

Moreover consider an ancilla which comprises component subsystems of the form

$$
\left|\Phi^{(M)}\right\rangle=\left|\Phi^{\left(M_{1}\right)}\right\rangle \otimes\left|\Phi^{\left(M_{2}\right)}\right\rangle
$$

where $M=M_{1}+M_{2}$. The requirement that $E_{\mathrm{P}}\left(\left|\Phi^{\prime(M)}\right\rangle\right)=0$ implies that $\left|\Phi^{(M)}\right\rangle$ can be expressed in the form

$$
\left|\Phi^{\prime(M)}\right\rangle=\sum_{m=0}^{M} c_{m}^{\prime}|\phi(m)\rangle_{\mathrm{A}} \otimes|\varphi(M-m)\rangle_{\mathrm{B}}
$$

where the tensor product form of Eq. (5.3) restricts the variability of the coefficients $c_{m}^{\prime}$. This is a further constraint on the coefficients, in addition to the normalization condition, and so the entanglement made accessible by a composite ancilla cannot exceed that given by a single component ancilla composed of a maximum of $\mathrm{M}$ particles. Hence the use of multiple ancillae in a tensor product state does not give any advantage in optimizing the accessible entanglement. 


\section{CONCLUSION}

Quantum reference frames are of particular theoretical interest and the phenomena of unlocking entanglement has proven to be a very interesting application. Our analytical techniques have proven to be fruitful in producing exact solutions in the case of a single shared particle and guidelines for sensible approximations for system states of large $N$. Much work is needed to produce exact analytical solutions for more general system states. However, the results extracted seem to establish the intuition that pure states with a broad particle-number distribution act as good reference frames for the particle number SSR. Indeed, the optimal reference state in the infinite-particle limit $M \rightarrow \infty$ makes all the entanglement in the single shared particle accessible.

\section{Acknowledgments}

This work was supported by the Australian Research Council and the Queensland State Government.

\section{APPENDIX A: SOLUTION AS A SERIES OF POLYNOMIALS IN $\beta$}

Here we explore the nature of the iterative solution of the recurrence relation Eq. (2.20) for the case where the system consists of a single particle which is equally shared between the sites. Setting $n=0$ and using the boundary condition $\left|c_{-1}\right|^{2}=0$ gives an expression for $\left|c_{1}\right|^{2}$ in terms of $\left|c_{0}\right|^{2}$. Next, by setting $n=1$ we find an expression for $\left|c_{1}\right|^{2}$ and so on. Continuing in this way yields a solution to Eq. (2.20) that is expressed in terms of just $\beta$ and $\left|c_{0}\right|^{2}$ as

$$
\left|c_{n}\right|^{2}=P_{n}(\beta)\left|c_{0}\right|^{2}
$$

where $P_{n}(\beta)$ are polynomials in $\beta$ of order $n$ given by

$$
\begin{aligned}
& P_{0}(\beta)=1 \\
& P_{1}(\beta)=\beta-1 \\
& P_{2}(\beta)=(\beta-1)(\beta-2) \\
& P_{3}(\beta)=\left(\beta^{2}-3 \beta+1\right)(\beta-2) \\
& P_{4}(\beta)=(\beta-3)(\beta-1)\left(\beta^{2}-3 \beta+1\right) \\
& P_{5}(\beta)=\left(\beta^{3}-5 \beta^{2}+6 \beta-1\right)(\beta-3)(\beta-1) \\
& P_{6}(\beta)=\left(\beta^{3}-6 \beta^{2}+10 \beta-4\right)\left(\beta^{3}-5 \beta^{2}+6 \beta-1\right)
\end{aligned}
$$

etc. Due to the normalization condition Eq. (2.7), we have the property that

$$
\sum_{n=0}^{M} P_{n}(\beta)=\frac{1}{\left|c_{0}\right|^{2}}
$$

Alternatively, rearranging the recurrence relation Eq. (2.19) as

$$
\left|c_{n-1}\right|^{2}=\frac{(\beta-1)\left|c_{n}\right|^{4}-\left|c_{n}\right|^{2}\left|c_{n+1}\right|^{2}}{\left|c_{n}\right|^{2}+\left|c_{n+1}\right|^{2}},
$$

leads to a different form of the solution. For this we set $n=M$ and use the boundary condition $c_{M+1}=0$ with Eq. A4 to get an expression for $\left|c_{M-1}\right|^{2}$ in terms of $\left|c_{M}\right|^{2}$. Continuing in this way with reducing values of $n$ we find that any coefficient $\left|c_{M-n}\right|^{2}$ can be written in terms of polynomials in $\beta$ and $\left|c_{M}\right|^{2}$. The symmetry of Eq. 2.19) with respect to interchanging $n$ with $m=$ $M-n$ ensures that the polynomials are the same as those appearing in Eq. (A1), i.e.

$$
\left|c_{M-n}\right|^{2}=P_{n}(\beta)\left|c_{M}\right|^{2} \text {. }
$$

Substituting $n=M$ into Eq. (A5) and (A1) yields

$$
\left|c_{0}\right|^{2}=P_{M}(\beta)\left|c_{M}\right|^{2},\left|c_{M}\right|^{2}=P_{M}(\beta)\left|c_{0}\right|^{2}
$$

respectively. The simultaneous solution to Eqs. (A6) is

$$
P_{M}(\beta)=1,\left|c_{0}\right|^{2}=\left|c_{M}\right|^{2}
$$

and so from Eqs. (A1) and (A5) we find that

$$
\left|c_{n}\right|^{2}=\left|c_{M-n}\right|^{2}
$$

for $n=0,1, \ldots, M$. Thus the solution which represents the optimal reference state is symmetric in this sense.

\section{APPENDIX B: PHASE PROPERTIES OF THE STATE $\left|\Phi_{1}^{(M)}\right\rangle_{\mathrm{AB}}$}

The unitary operator $\hat{U}_{\mathrm{A}}=e^{i \hat{N}_{\mathrm{A}}\left(\hat{N}_{\mathrm{A}}-1\right) \vartheta}$ in the definition of the state $\left|\Phi_{1}^{(M)}\right\rangle_{\mathrm{AB}}$ in Eq. (??) represents a Kerrlike nonlinear interaction. The action of this operator on coherent states has been discussed extensively in the literature (see e.g. Refs. [30, 31, 32, 33, 34]). However, in our case the operator $\hat{U}_{\mathrm{A}}$ acts on the shared phase state $\left|\Phi_{0}^{(M)} 1\right\rangle_{\mathrm{AB}}$ in Eq. (??) and so requires a minor extension of previous work. For this we use a previous result to derive a form of the operator $\hat{U}_{\mathrm{A}}$ that allows us to infer the pertinent phase properties of $\left|\Phi_{1}^{(M)}\right\rangle_{\mathrm{AB}}$.

One of us has previously shown that [34]

$$
\begin{gathered}
\sum_{k=0}^{K-1} c_{k} \exp \left(i n \phi_{k}\right)=\exp [-i \vartheta n(n-1)] \\
c_{j}=\frac{1}{K} \sum_{n=0}^{K-1} \exp [-i \vartheta n(n-1)] \exp \left(i n \phi_{j}\right)
\end{gathered}
$$

where

$$
\begin{aligned}
\vartheta & =\frac{\pi J}{K} \\
\phi_{k} & =\phi_{0}+\frac{2 \pi k}{K} \\
\phi_{0} & =\{[J(K-1)] \bmod 2\} \frac{\pi}{K}
\end{aligned}
$$


and $J$ and $K$ are positive integers sharing no common factors. Expanding $\hat{U}$ in terms of the Fock basis then yields

$$
\begin{aligned}
\hat{U}_{\mathrm{A}} & =\sum_{n=0}^{\infty} \exp [-i \vartheta n(n-1)]|n\rangle_{\mathrm{AA}}\langle n| \\
& =\sum_{n=0}^{\infty} \sum_{k=0}^{K-1} c_{k} \exp \left(i n \phi_{k}\right)|n\rangle_{\mathrm{AA}}\langle n| \\
& =\sum_{k=0}^{K-1} c_{k} \exp \left(i \hat{N}_{\mathrm{A}} \phi_{k}\right)
\end{aligned}
$$

which is in the form of a series of phase shifting operators $\exp \left(i \hat{N} \phi_{k}\right)$. The case discussed in the text is for the choice $J=1$ and $K=2$ for which $\vartheta=\pi / 2, \phi_{0}=\pi / 2$, $\phi_{1}=3 \pi / 2, c_{0}=(1+i) / 2$, and $c_{1}=(1-i) / 2$, and so

$$
\begin{aligned}
\left|\Phi_{1}^{(M)}\right\rangle_{\mathrm{AB}}= & \hat{U}_{\mathrm{A}}\left|\Phi_{0}^{(M)}\right\rangle_{\mathrm{AB}} \\
= & \frac{1+i}{2 \sqrt{M+1}} \sum_{n=0}^{M} e^{i n \pi / 2}|n, M-n\rangle_{\mathrm{AB}} \\
& +\frac{1-i}{2 \sqrt{M+1}} \sum_{n=0}^{M} e^{-i n \pi / 2}|n, M-n\rangle_{\mathrm{AB}}
\end{aligned}
$$

Using Eq. (2.50) then shows that Eq. (??) is the phase difference probability density corresponding to this state.
[1] G. C. Wick, A. S. Wightman, and E. P. Wigner, Phys. Rev. 88, 102 (1952).

[2] Y. Aharanov and L. Susskind, Phys. Rev. 155, 1428 (1967).

[3] F. Girelli and D. Poulin, J. Phys. Conf. Ser. 68, 012025 (2007).

[4] F. Girelli and D. Poulin, arXiv:0710.4393v1 (2007).

[5] J. A. Vaccaro, F. Anselmi, H. M. Wiseman, and K. Jacobs, Phys. Rev. A 77, 032114 (2008).

[6] S. D. Bartlett and H. M. Wiseman, Phys. Rev. Lett. 91, 097903 (2003).

[7] H. M. Wiseman and J. A. Vaccaro, Phys. Rev. Lett. 91, 097902 (2003).

[8] J. A. Vaccaro, F. Anselmi, and H. M. Wiseman, Inter. J. Quant. Inf. 1, 427 (2003).

[9] S. D. Bartlett, T. Rudolph, and R. W. Spekkens, 79, 555 (2007).

[10] S. D. Bartlett, T. Rudolph, and R. W. Spekkens, Phys. Rev. Lett. 91, 027901 (2003).

[11] S. D. Bartlett, T. Rudolph, and R. W. Spekkens, Phys. Rev. A 70, 032307 (2004).

[12] E. Bagan, S. Iblisdir, and R. Munoz-Tapia, Phys. Rev. A 73, 022341 (2006).

[13] N. H. Linder, P. F. Scudo, and D. Bruß, Inter. J. Quant. Inf. 4, 131 (2006).

[14] S. J. van Enk and H. J. Kimble, Quant. Inf. Comp. 2, 1 (2001).

[15] J. Gea-Banacloche, Phys. Rev. A 65, 022308 (2002).

[16] W. M. Itano, Phys. Rev. A 68, 046301 (2003).

[17] H. Nha and H. J. Carmichael, Phys. Rev. A 71, 013805 (2005).

[18] M. Ozawa, Int. J. Quant. Inf. 1, 569 (2003).

[19] M. Ozawa, "Universal Uncertainty Principle and Quantum State Control under Conservation Laws" in S.M. Barnett, E. Anderson, J. Jeffers, P. Öhberg, O. Hirota, eds., Proceedings of Seventh International Conference on Quantum Communication, Measurement and Computing (QCMC 2004), Glasgow, UK, 25 Jul 2004 - 29 Jul 2004, AIP Conf. Proc. 734, 95 (2004).

[20] M. Ozawa, Phys. Lett. A 320, 367 (2004).

[21] J. Gea-Banacloche and M. Ozawa, J. Opt. B. 7, S326 (2005).

[22] S. J. Jones, H. M. Wiseman, S. D. Bartlett, and J. A.
Vaccaro, Phys. Rev. A 74, 062313 (2006).

[23] M. R. Dowling, A. C. Doherty, and H. M. Wiseman, Phys. Rev. A 73, 052323 (2006).

[24] D. T. Pegg and S. M. Barnett, Europhys. Lett. 6, 483 (1988).

[25] J. A. Vaccaro and D. T. Pegg, J. Mod. Optics 37, 17 (1990).

[26] S. M. Barnett and J. A. Vaccaro, eds., The Quantum Phase Operator: A Review (Taylor and Francis, London, 2007).

[27] G. S. Summy and D. T. Pegg, Opt. Comm. 77, 75 (1990).

[28] D. W. Berry and H. M. Wiseman, Phys. Rev. Lett. 85, 5098 (2000).

[29] D. Stoler, B. E. A. Saleh, and M. C. Teich, Opt Acta 32, 345 (1985).

[30] G. J. Milburn, Phys. Rev. A 33, 674 (1986).

[31] Z. Bialynicka-Birula, Phys. Rev. 173, 1207 (1968).

[32] R. T. A. Miranowicz and S. Kielich, Quantum Optics 253, 2 (1990).

[33] M. Paprzycka and R. Tanas, 4, 331 (1992).

[34] J. Vaccaro and A. Orlowski, Phys. Rev. A 51, 4172 (1995).

[35] S. van Enk, Phys. Rev. A 71, 032339 (2005).

[36] Our definition of finite reference systems differs from the bounded reference systems of Bartlett et al. [9]. In the latter case the bound is on some physical parameter such as the number of spin $1 / 2$ particles or the mean photon number. In particular, bounded reference systems can have either finite or infinite dimensional Hilbert spaces. In contrast finite reference systems are restricted by having a finite dimensional Hilbert space.

[37] We note that Jones et al. [22] use the term extractable entanglement in place of accessible entanglement.

[38] Our constraint of a fixed number of particles means that it is not possible to make a direct comparison between the reference states considered in this paper and van Enks refbit state [35] which is based on an uncertain number of shared particles.

[39] In contrast, the state $|n\rangle$ is invariant to the group operation $\hat{T}(\phi)$ in the sense that $\hat{T}(\phi)|n\rangle\left\langle n\left|\hat{T}^{\dagger}(\phi)=\right| n\right\rangle\langle n|$, which illustrates the symmetry of $|n\rangle$ with respect to $\mathrm{U}(1)$ [5]. 